\title{
Assessing workforce needs for the emerging CAR-T cell therapy industry
}

\author{
The complicated and labor-intensive manufacturing of novel cell and gene therapies requires efforts to improve \\ workforce development programs for the nascent cell therapy industry broadly, and for cell manufacturing \\ more specifically.
}

nvestment in cell and gene therapies is growing rapidly as key proof-of-concept therapies have gained regulatory approval and market access around the world. These include chimeric antigen receptor $\mathrm{T}$ cell (CAR-T) therapies for some forms of leukemia and lymphoma (tisagenlecleucel, axicabtagene ciloleucel, brexucabtagene autoleucel and lisocabtagene maraleucel) and multiple myeloma (for example, idecabtagene vicleucel) as well as gene therapies for pediatric spinal muscular atrophy (onasemnogene abeparvovec-xioi) and retinal dystrophy (voretigene neparvovec-rzyl). By 2025, the US Food and Drug Administration (FDA) expects to approve 10 to 20 cell and gene therapies annually ${ }^{1}$. However, development and, especially, manufacturing of these novel therapies is complicated and labor-intensive ${ }^{2,3}$, which raises concerns that the lack of a skilled workforce may hinder growth of this field - potentially slowing development, raising costs and limiting the availability of novel therapies. As a result, workforce development has been highlighted as a key challenge for the industry ${ }^{4-7}$ and a number of workforce development initiatives are in progress.

To inform and support these efforts, it is critical to better understand the workforce needs for cell therapy, including the specific skills that cell therapy developers and manufacturers require as well as the training that they prioritize in their recruiting. Here we examine workforce needs for three leading CAR-T cell therapy companies: Bristol Myers Squibb, Gilead Sciences and Novartis. We focus on CAR-T cell therapy because it is a recently commercialized combined cell and gene therapy, with FDA approvals for five products in the USA since August 2017. In particular, drawing on methods used in characterizations of the broader biotechnology and pharmaceutical industries ${ }^{8,9}$, we focus on job advertisements posted by three companies with approved CAR-T cell therapy products and ongoing CAR-T cell therapy development. While cell therapy is a broad field, we believe focusing our assessment on companies with recently commercialized products will provide a fuller picture of job needs, including not only the R\&D prioritized by early-stage companies but also manufacturing, marketing and other roles that may increase in importance as a product nears or gains market access. Our results should be generally applicable to other cell and combined cell and gene therapies that are currently in development, and inform curriculum development and training efforts for this nascent industry.

\section{Methods}

We collected job advertisements from the official websites of each of the three companies (https://careers.bms.com/ jobs, https://gilead.wd1.myworkdayjobs. com/kitepharmacareers and https://www. novartis.com/careers/career-search), searching specifically for advertisements that contained the term 'CAR-T'. We chose this term because our pilot searches indicated that it primarily captured jobs that included work on CAR-T cell therapies as a key component of the position's responsibilities. By contrast, broader terms such as 'cell therapy' captured CAR-T cell therapy jobs as well as a range of other jobs that were not clearly related to CAR-T cell therapy.

We conducted the same searches twice, first in August 2019 (22 August 2019 to 23 August 2019) and again in October 2020 (27 October 2020 to 29 October 2020), to understand the extent to which workforce needs persisted or changed over time. Our second search occurred well into the COVID-19 pandemic, so our data and analysis provide insight into the extent to which CAR-T cell therapy firms continued to advertise for and potentially make new hires in this complicated and atypical environment. When advertisements clearly sought to fill several distinct positions (for example, the same advertisement might aim to recruit both a 'Director of Marketing' and an 'Assistant Director of Marketing'), we counted them one time for each position. We also excluded a small number of advertisements posted in languages other than English. In the few cases in which the same position title was posted several times (for example, Bristol Myers Squibb posted several 'Lead Manufacturing Associate' positions), we retained these as distinct advertisements, assuming the firm was seeking to hire several candidates into this role. This yielded a final dataset of 326 advertisements (123 from our August 2019 searches and 203 from our October 2020 searches) (Table 1).

To understand workforce needs, we reviewed each job posted in our August 2019 dataset and manually coded a series of general characteristics (such as company, job title, job classification and geographical location of job) and educational requirements (such as minimum degree required, years of experience required and discipline required). We focused on minimum educational credentials as they represent the standard that training programs must meet to align with typical industry entry points - although we note that some advertisements specified several desired educational levels, with, for example, less experience required for candidates with more education. Two authors (L.D.H. and A.D.L.) developed the initial codebook through review of a randomly selected subset of 15 job advertisements. Following codebook development, a single coder (L.D.H.) initially coded the remaining August 2019 advertisements. Similarly, a single coder (H.L.R.) initially coded the October 2020 advertisements. Most coding was straightforward, but ambiguous cases were flagged for discussion and resolved by consensus.

\section{Results}

On the basis of the August 2019

dataset of 123 CAR-T cell-therapy job advertisement, the most common minimum educational level specified was a four-year undergraduate degree, identified in $71 \%$ of advertisements (Table 2). By contrast, only $8 \%$ of advertisements specified lower educational requirements (for example, 
Table 1 | Number of CAR-T job advertisements by company

\begin{tabular}{lllll} 
& Bristol Myers Squibb & Gilead Sciences & Novartis & Total \\
\hline August 2019 & $87(71 \%)$ & $29(24 \%)$ & $7(6 \%)$ & 123 \\
October 2020 & $168(83 \%)$ & $19(9 \%)$ & $16(8 \%)$ & 203
\end{tabular}

Table 2 | Minimum education level by advertisement for 2019 CAR-T job advertisements

\begin{tabular}{lllll} 
& Bristol Myers Squibb & Gilead Sciences & Novartis & Total \\
\hline Less than bachelor's & $10(11 \%)$ & 0 & 0 & $10(8 \%)$ \\
Bachelor's & $65(75 \%)$ & $18(62 \%)$ & $4(57 \%)$ & $87(71 \%)$ \\
Master's $^{2}$ & $1(1 \%)$ & $1(3 \%)$ & $2(29 \%)$ & $4(3 \%)$ \\
Doctoral $^{\mathrm{a}}$ & $8(9 \%)$ & $8(28 \%)$ & $1(14 \%)$ & $17(14 \%)$ \\
Not specified $_{\text {Total }}$ & $3(3 \%)$ & $2(7 \%)$ & 0 & $5(4 \%)$ \\
\hline
\end{tabular}

alncludes $\mathrm{PhD}, \mathrm{MD}$ and other doctoral degrees.

high school diploma, General Educational Development test or two-year degree). Approximately $14 \%$ of advertisements required $\mathrm{PhDs}$, MDs or other doctoral-level training.

The job advertisements covered the entire value chain for CAR-T cell therapy. Bristol Myers Squibb included a 'job classification' in each of the 87 advertisements in August 2019. The most common classification was 'Technical development', which accounted for $54 \%$ of advertisements. This category included positions focused on manufacturing, production planning, process engineering and similar roles. The next most-common categories were 'Quality' (14\%), 'Sales and marketing' (14\%), 'Research and early development' (6\%) and 'Medical' (5\%). Although the job classifications changed slightly, this pattern was similar in the October 2020 advertisements, with 'Manufacturing/ ops' (27\%) and 'Engineering' (21\%) both of which were probably classified as 'Technical development' in the previous year - combining to account for $48 \%$ of advertisements, followed by 'Quality assurance methods' (15\%). Job advertisements from Novartis and Gilead Sciences had similar position titles, although these companies posted fewer advertisements and used different position classifications, which makes direct comparisons difficult.

Given the unique nature of cell therapy manufacturing with all currently approved CAR-T cell therapies produced on a patient-specific basis, we examined advertisements specifically including 'manufacturing' in the job title in more detail (Table 3). In all, 50 advertisements (15\%) (17 from the August 2019 data collection and 33 from the October 2020 data collection) included 'manufacturing' in their job title. These ranged from manufacturing technician and associate roles to more senior managerial roles. In both years, variations (for example, senior, lead and pm (that is, night shift)) on the manufacturing associate role accounted for a large share of the advertisements (12 out of $17(71 \%)$ in 2019 , and 14 out of 33 $(42 \%)$ in 2020). Given the individualized nature of CAR-T cell production and the need to scale-out rather than scale-up in this field, basic manufacturing roles such as manufacturing technicians and manufacturing associates - are likely to be in high demand as the CAR-T cell therapy industry grows. Table 3 provides an overview of the expected responsibilities and desired qualifications for these roles to help to inform ongoing and future workforce development efforts. On the basis of the job advertisements we analyzed, both the manufacturing technician and the manufacturing associate role appear open to candidates with minimal or (in some cases) no direct work experience in cell therapy and manufacturing. For the technician role (an entry-level position appropriate for graduates of two-year programs), experience working in a regulated environment is desired, but some of this experience could potentially be gained during laboratory courses, in internships or from work in other regulated fields outside of cell therapy. For the manufacturing associate role (an entry-level manufacturing position primarily for graduates of four-year programs), more field-specific knowledge (for example, FDA guidance or current good manufacturing practice regulations) is expected and cell manufacturing experience, including cell culture experience, is preferred, but (at least in some cases) not required. Candidates for both roles would benefit from relevant laboratory and/or internship experience integrated into their academic programs.

\section{Discussion}

Our data collection and analysis identified active recruiting by three leading CAR-T cell therapy companies in 2019 that continued in 2020 amid the still-ongoing COVID19 pandemic. Additionally, a majority of advertisements at Bristol Myers Squibb, the company that posted the largest number of advertisements, focused on technical jobs, lending support to calls for improved training programs for biomanufacturing and cell manufacturing ${ }^{4}$. Publicly announced plans by key CAR-T cell therapy companies align with our analysis of posted job advertisements. For example, Bristol Myers Squibb is building a new cell therapy manufacturing facility in Devens, $\mathrm{MA}$, and has estimated that it will require "several hundred new employees" in the coming years ${ }^{10}$. Similarly, Kite Pharma (the CAR-T cell subsidiary of Gilead Sciences) is building a manufacturing facility in Frederick, Maryland that will "employ a few hundred people over the next several years" 11 .

Meeting the demand for new employees in the growing cell and gene therapy industry and, especially, for manufacturing these labor-intensive therapies will require a substantial effort engaging technical colleges, four-year universities, and scientific and industry groups.

Several of these training programs are now in development or underway. These include programs supported by major federal investments in the USA, such as the National Institute for Innovation in Manufacturing Biopharmaceuticals (NIIMBL) (https://niimbl.force.com/s/), part of the Manufacturing USA network of manufacturing innovation institutes, and the National Science Foundation-funded Engineering Research Center for Cell Manufacturing Technologies (CMaT) (www. cellmanufacturingusa.org). For example, NIIMBL awards grants to its academic and industry members to develop, pilot and implement training programs to help to meet industry workforce needs. Similarly, CMaT develops new curricular materials for use at its core institutions and with its educational partners (such as local school systems or technical colleges), and pilot-tests them. 
Table 3 | Expectations for entry-level CAR-T therapy manufacturing roles

\begin{tabular}{|c|c|c|}
\hline & Manufacturing technician & Manufacturing associate \\
\hline Example duties & $\begin{array}{l}\text { Supports cellular therapeutic } \\
\text { manufacturing and logistics by: } \\
\text { - performing operations } \\
\text { described in SOPs and batch } \\
\text { records } \\
\text { - completing documentation } \\
\text { - executing daily support } \\
\text { operations (for example, } \\
\text { materials kitting, stocking and } \\
\text { maintenance) }\end{array}$ & $\begin{array}{l}\text { Manufactures cellular therapeutic agents (for } \\
\text { example, human-blood-derived components) } \\
\text { by: } \\
\text { - performing patient process unit operations } \\
\text { described in SOPs and batch records } \\
\text { - completing documentation } \\
\text { - writing manufacturing operating procedures }\end{array}$ \\
\hline Education & $\begin{array}{l}\text { Associate's degree or a year of } \\
\text { experience }\end{array}$ & $\begin{array}{l}\text { Bachelor's degree in relevant field or equivalent } \\
\text { work experience } \\
\text { Some roles may be open to high-school } \\
\text { graduates or associate's-degree holders with } \\
\text { relevant experience }\end{array}$ \\
\hline Work experience & $\begin{array}{l}\text { Six months in regulated/Good } \\
\text { Practice environment }\end{array}$ & $\begin{array}{l}\text { CGMP cell-manufacturing experience preferred, } \\
\text { but none required for entry-level position }\end{array}$ \\
\hline Desired skills & Good documentation practices & $\begin{array}{l}\text { Knowledge of relevant CGMP regulations and } \\
\text { FDA guidance } \\
\text { Aseptic processing techniques } \\
\text { Precautions for handling human-derived } \\
\text { materials in BSL-2 } \\
\text { Cell expansion } \\
\text { Cell washing } \\
\text { Cell separation } \\
\text { Cryopreservation }\end{array}$ \\
\hline
\end{tabular}

BSL-2, biosafety level 2; CGMP, current good manufacturing practice; SOPs, standard operating procedures.

In some cases, individual universities have developed programs that are specific to cell and gene therapy or have added tracks to existing programs. For example, the New Jersey Institute of Technology offers a professional master's degree in cell and gene therapy sciences as an option for students enrolled in its Master of Science in Pharmaceutical Chemistry program. Similarly, the University of Georgia offers a Biopharmaceuticals and Cell Manufacturing track in its Master of Biomanufacturing and Bioprocessing program. In addition to degree programs, some institutions offer shorter certificates. The Cellular Therapies Certificate Program offered by the American Association of Blood Banks in cooperation with George Washington University is one such example. This program is a self-paced series of twelve modules recorded by George Washington University faculty and other selected experts in the field, and is designed to provide an introduction to cell therapies.

Efforts to prepare the cell manufacturing workforce are underway in Europe as well. The UK's Cell and Gene Therapy Catapult offers an advanced therapy apprenticeship program that places students at cell and gene therapy companies while they complete their studies. In Switzerland, the Biofactory
Competence Center (a not-for-profit company) offers a range of cell and gene therapy-specific trainings.

Despite these and other similar efforts to develop and implement cell therapy training programs, most programs are small in scale and will probably need to grow or be complemented by additional efforts for workforce needs to be adequately addressed. Furthermore, the potential for duplication among various programs is high and coordination will be necessary. The development of workforce training programs for cell and gene therapies is complicated by several factors, including the rapidly changing technology and the regional nature of key labor markets (especially for entry-level manufacturing positions). We believe that the analysis presented here - examining the number and type of job advertisements posted, the minimum education expected and, for manufacturing roles, the key skills expected - will be useful to both those seeking to understand the state of the cell and gene therapy industry, and those actively developing programs to help to meet future workforce needs.

Our study is subject to several limitations. Our data collection effort did not generate a comprehensive set of CAR-T cell job advertisements nor do we have reason to believe it is a representative selection of all CAR-T cell therapy job advertisements. Rather, we deliberately selected companies with products on the market to get a sense of what near-term workforce needs might look like as the industry matures and more products gain market access. In addition, our search strategy may have excluded some relevant job advertisements that did not mention CAR-T cells specifically (although we expect this is a relatively small number). Furthermore, the format and content of the various job advertisements we collected and analyzed were not consistent either within or across companies, which complicated the coding effort. Finally, the relationship between the number of job advertisements that a company places and the number of positions that it seeks to fill is not precisely known. For example, we cannot always tell whether a company seeks to hire several 'Marketing Associates' or 'QC Technicians' from a single job advertisement. This suggests that our analysis may underestimate workforce needs, especially for entry- and lower-level roles for which several candidates could be hired from a single job advertisement. Despite these limitations, our analysis provides one of the first assessments of workforce needs in the emerging CAR-T cell therapy industry and should prove useful to academic centers and industry groups that are developing strategies to meet this challenge.

\section{Linda D. Ho ${ }^{1,2}$, Hadassah L. Robbins ${ }^{1,2}$ and Aaron D. Levine (D) 1,2,3凶 \\ ${ }^{1}$ School of Public Policy, Georgia Institute of Technology, Atlanta, GA, USA. ${ }^{2}$ NSF Engineering Research Center for Cell Manufacturing Technologies (CMaT), Atlanta, GA, USA. ${ }^{3}$ Parker H. Petit Institute for Bioengineering and Bioscience, Georgia Institute of Technology, Atlanta, GA, USA.

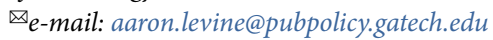

Published online: 15 February 2022 https://doi.org/10.1038/s41587-022-01212-6

References

1. Gottlieb, S. Statement from FDA Commissioner Scott Gottlieb, M.D. and Peter Marks, M.D., Ph.D., Director of the Center for Biologics Evaluation and Research on new policies to advance development of safe and effective cell and gene therapies. fda.gov, https://go.nature.com/31Orwue (January 2019).

2. Dodson, B. P. \& Levine, A. D. BMC Biotechnol. 15, 70 (2015).

3. Levine, B. L., Miskin, J., Wonnacott, K. \& Keir, C. Mol. Ther Methods Clin. Dev. 4, 92-101 (2017).

4. National Cell Manufacturing Consortium. Achieving Large-Scale, Cost-Effective, Reproducible Manufacturing of High-Quality Cells: A Technology Roadmap to 2025 (Nexight Group, 2016); https://cellmanufacturingusa.org/sites/default/files/NCMC Roadmap 021816 high res-2.pdf

5. Sullivan, J. As companies dump money into manufacturing, where will they find the trained workers to man the lines? Endpoints News, https://go.nature.com/3FoeEsu (June 2021).

6. McNiece, I. K., Wacker, K. K., Kurtzberg, J. \& Warkentin, P. I. Cytotherapy 23, 886-893 (2021). 
7. Eastman, P. Oncol. Times 41, 30-31 (2019).

8. Lindburg, L., Bozinovic, L. \& Susek, R. Nat. Biotechnol. 37, 481-483 (2019).

9. Mason, J. L. et al. FASEB J. 30, 2673-2683 (2016).

10. Squibb B. M. New state-of-the-art cell therapy manufacturing facility under construction to expand Bristol Myers Squibb global footprint. news.bms.com, https://go.nature.com/3K1Guyh (February 2021).

11. Keown, A. Kite, a Gilead company, looks to expand its cell therapy Maryland operations. BioSpace, https://go.nature. com/3I0MYeS (August 2020).

\section{Acknowledgements}

This material is based upon work supported by the National Science Foundation under grant no. EEC-1648035. The authors thank A. Incorvaia for helpful discussions early in the development of this project, and members of the CMaT Supply Chain team for helpful discussions and support. Bristol Myers Squibb is a CMaT industry partner, but employees of Bristol Myers Squibb did not have any role in the conceptualization, design, data collection, analysis or preparation of this manuscript.
Author contributions

L.D.H. collected data in 2019, analyzed data and contributed to the first draft of the manuscript. H.L.R collected data in 2020 , analyzed data and contributed to the first draft of the manuscript. A.D.L. designed the project, oversaw data collection and development of the analysis strategy and revised and finalized the manuscript.

Competing interests

The authors declare no competing interests. 